\title{
Triple Diffusive MHD Casson Fluid Flow Over a Vertical Wall with Convective Boundary Conditions
}

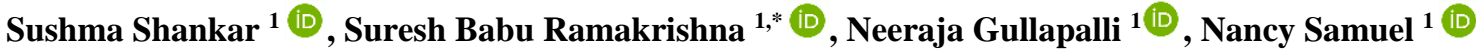 \\ 1 Department of Mathematics, M S Ramaiah Institute of Technology, Bangalore-560 054, Karnataka, India; \\ sushmsrit@gmail.com (S.S.); sureshbabu_r80@yahoo.co.in (S.B.R.); gneerajamaths@gmail.com (N.G.); \\ samuelnancy1984@gmail.com (N.S.); \\ * Correspondence: sureshbabu_r80@yahoo.co.in;
}

Scopus Author ID 57199323358

Received: 12.01.2021; Revised: 7.02.2021; Accepted: 10.02.2021; Published: 17.02.2021

\begin{abstract}
In this article, we study, effects of triple diffusive on a mixed convective viscous flow of an MHD Casson fluid through a vertical permeable wall numerically with convective BC's. The governing equations are modeled and derived for the triple diffusive boundary layer flow to examine the fluid's nature under the influence of thermal conductivity and solutal diffusivity. Using an effective and suitable similarity transformation, highly non-linear coupled PDE's are reduced to a series of coupled ODE's and are solved by the Shooting technique with the help of the integral scheme of Runge KuttaFehlberg. To know the fluid properties' behavior, a numerical computation has been carried out for the non-dimensional parameters, which control the flow and demonstrated through plots such as permeability, convective parameter, Casson parameter, and buoyancy ratio parameters of the physical system. In the absence of a few non-dimensional parameters, present findings are compared with previously published work to validate our numerical scheme and found to be in good agreement with up to six decimal places of accuracy.
\end{abstract}

Keywords: triple diffusive; MHD; Casson fluid; mixed convection; vertical wall; shooting technique.

(C) 2021 by the authors. This article is an open-access article distributed under the terms and conditions of the Creative Commons Attribution (CC BY) license (https://creativecommons.org/licenses/by/4.0/).

\section{Introduction}

The study of heat and mass transfer received considerable interest in many theoretical and experimental/practical aspects because of its various applications in industry, scientific, and engineering processes. In the literature, such problems are dealt with the Newtonian/nonNewtonian and non-Darcian fluids through/past a vertical surface with different boundary conditions. References and comprehensive literature surveys related to porous medium have been described by Nield and Bejan [1]. The advancement of modern technologies has demanded the study of fluid streams, which encompass the interaction of numerous phenomena. Double diffusion appears in buoyancy compelled flow due to the amalgamation of temperature and concentration gradients; many authors had studied such a study in a porous medium. Kumari et al. [2] have been studied mixed convection flow under the influence of the magnetic field with an isotropic solid matrix and dissipation. Boussinesq approximations are tested by Mamou et al. [3] for a double-diffusive natural convective flow of a Darcy model in an inclined porous layer. The boundary layer flow of a sloping plate with IHG and chemical reaction in a saturated porous medium by Patil et al. [4], and the studied under the impact of Soret effect by Murthy et al. [5]. Mallikarjuna et al. [6] examined non-uniform heat sources' effects on a rotary device over a plate fixed in a non-Darcy porous medium. The results of 
double-diffusive on mixed convective viscous fluid through a plate with variable fluid properties were discussed by Suresh Babu et al. [7].

However, suppose there are gradients of more than two diffusing elements that exist. In that case, the species concentration and temperature are known as multiple diffusive convection. The presence of more than one chemical dissolved in a liquid mixture describes common phenomena such as transport of toxic elements, latent water flow, and acid flow. Triple diffusion comprises three constituents with different diffusivities. Application of triple diffusive convection can be noticed in the modeling of medical airing tool for the study of anomalies in fatty acid, encompassing numerous constituents such as saturated fat (Cholesterol-low-density lipoproteins, high-density lipoproteins) and triglycerides, which retain dissimilar diffusivities. However, triple diffusion in a non-Newtonian fluid has practical applications in many medical sciences and bioengineering. For example, aqueous suspensions of deoxyribonuc DNA contain more than two independently diffusing constituents with dissimilar diffusivities found in the book by Rionero et al. [8]. The triple diffusion in a Newtonian or non-Newtonian fluid has been studied extensively for a flat plate with convective boundary conditions by Khan et al. [9], stability analysis of a viscoelastic fluid by Raghunatha et al. [10], NaCl-water and sucrose water solutions by Patil et al. [11, 12], along with a stretching sheet with variable thickness by Nancy Samuel [13], for a duct by Umavathi et al. [14], linear stability analysis of a Maxwell fluid by Raghunatha et al. [15], for various porous cavities by Khan et al. [16], entropy generation analysis by Khan et al. [17] and diffusion of species using tangent hyperbolic rheology by Nawaz et al. [18].

Lateral inequality occurs in the direction of the deposited charge, and the magnetic fields applied between the opposite positions (perpendicular to the charge flow) induce currents of perpendicular, magnetic, and electrical currents to both magnetic fields. An electrically conducting fluid with an external constraint of the magnetic field can be regulated. The transfer rate can also be controlled. With this point of view, various industrial applications can be seen in many branches of sciences and technology like cooling nuclear reactors, boundary layer control in aerodynamics, plasma studies, petroleum industries, and crystal growth, etc. Hence, the authors are received new attention for the study of most general contexts of magnetohydrodynamics (MHD) with the external forces of an electrically conducting magnetic field. Casson fluid is a non-Newtonian fluid that exhibits stress on yield and requires high temperature for industrial and engineering problems. Isa et al. [19] investigated numerically MHD mixed convection flow over a stretching sheet. Sharada et al. [20] examined Soret, Dufour, radiation, and chemical reaction effects on MHD flow of a Casson fluid over an exponentially stretching surface. By considering convective boundary conditions, Hayat et al. [21] studied the stagnation point flow of a Casson fluid for stretching surface and John et al. [22] MHD Casson fluid over a plate with Joule heating. Srinivasa et al. [23] and Saqib et al. [26] examined numerically unsteady MHD Casson fluid flow over a vertical surface with an angle of inclination, constant heat flux, chemical reaction, whereas Muthuraj et al. [24] examined for stretching walls. Kataria et al. [25] studied heat, mass transfer characteristics of a MHD Casson fluid over an oscillating plate with ramped wall temperature. Heat transfer study of the unstable MHD Casson fluid over a plate with arbitrary wall shear and the constant temperature was analyzed by Khan et al. [27]. Vijaya Bhaskar et al. [28] investigated numerical analysis of MHD Casson fluid flow over an exponentially moving plate with a ramped wall temperature and surface concentration. Under the influence of thermal radiation and heat source/sink parameters along with the slip parameters, Sumera et al. [29] studied 2D heat 
transfer flow of a Casson-dependent nanofluid over a vertical stretching and shrinking sheet. The non-linear thermal radiated triple diffuse flow of a Casson nanofluid along a horizontal plate was studied by Manjappa et al. [30] and is theoretically investigated in the presence of more than one solution diffused in mixtures of fluids. Rao et al. [31] studied 3D heat mass transfer MHD motion over a stretching surface with Brownian flow, thermophoresis, and Dufour effects and found that the transfer rate of energy and concentration in the Newtonian fluid is relatively low compared to the non-Newtonian fluid. Computational analysis has been done by Lund et al. [32] on MHD micropolar fluid on a shrinking surface under the Joule effect and viscous dissipation. Ramanna et al. [33] investigated triple diffusion encoding MRI to measure the intra, and extra axonal diffusion tensors.

According to the authors' knowledge and the above literature, no research has been carried out to examine the effect of triple diffusion on an MHD Casson fluid with convective boundary conditions. However, the heat and mass diffusion components are always coupled in most real-world problems. This fact motivates us to study the joint effects of thermal and mass diffusion behavior on MHD mixed convective flow of a Casson fluid with convective boundary conditions in the boundary layer. Such a study has notable applications in real-world problems to enhance scientific expertise, develop new technologies, strengthen security, safety, and contribute significantly to health. It also has further applications in the fields of transport, biomedical, chemical engineering, paper production, microelectronics, and biomechanics, as well as industrialized methods such as metal spinning, nuclear squandering, polymer extrusion, refining of waste plastic, transpiration cooling process, the oil recovery process, etc.

\section{Materials and Methods}

\subsection{Mathematical formulation.}

Consider a Steady two-dimensional MHD mixed convective flow of a Casson fluid with a constant free stream velocity $U_{\infty}$ and temperature $T_{\infty}$ over a static permeable flat surface is considered. The $x$-axis is measured upwards along with the vertical plate, and $y$-axis is exactly normal(perpendicular) to it as shown in the problem's geometry and $u, v$ be the corresponding velocity components.

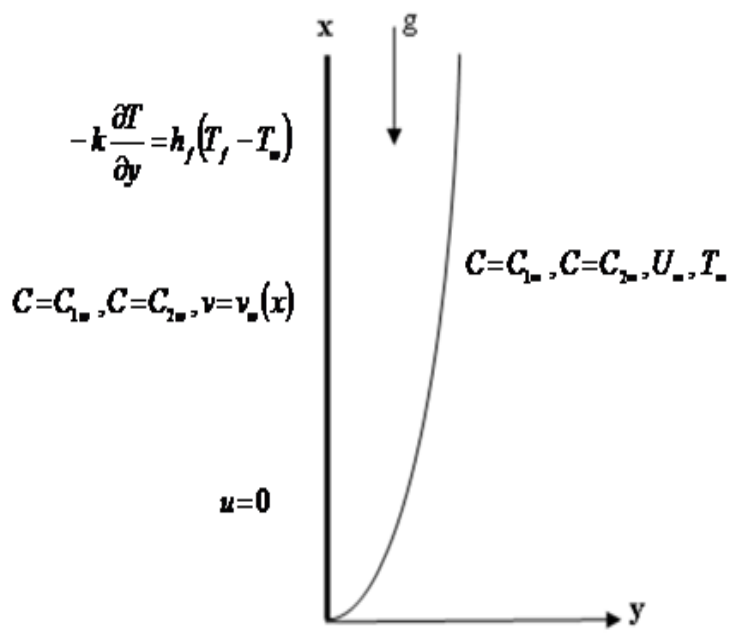

Figure 1. Physical model. 
It is assumed that two distinct chemical elements $S_{m}(m=1,2)$ having a concentration $C_{m}(m=1,2)$ are dissolved in the solution. Also, the left side of the plate is maintained with a constant fluid temperature $T_{f}$, which offers a variable heat transfer $h_{f}$. Assisting flow corresponds to the plate being heated by the fluid $\left(T_{f}>T_{\infty}\right)$, and opposing flow corresponds to the plate being cooled by the fluid $\left(T_{f}<T_{\infty}\right)$. The buoyancy approximation is taken into account and is coupled with the flow field. A moving magnetic field $B(x)$ is imposed normally on the surface. The induced magnetic field is not considered due to the very low magnetic Reynolds number when comparing the imposed magnetic field.

Under these assumptions, the basic governing PDE's equations are:

$\frac{\partial u}{\partial x}+\frac{\partial v}{\partial y}=0$

$u \frac{\partial u}{\partial x}+v \frac{\partial u}{\partial y}=v\left(1+\frac{1}{\beta}\right) \frac{\partial^{2} u}{\partial y^{2}}-\frac{\sigma}{\rho}[B(x)]^{2} u+g\left[\beta_{T}\left(T-T_{\infty}\right)+\beta_{C_{1}}\left(C_{1}-C_{1 \infty}\right)+\beta_{C_{2}}\left(C_{2}-C_{2 \infty}\right)\right]$,

$u \frac{\partial T}{\partial x}+v \frac{\partial T}{\partial y}=\alpha \frac{\partial^{2} T}{\partial y^{2}}$

$u \frac{\partial C_{1}}{\partial x}+v \frac{\partial C_{1}}{\partial y}=D_{S_{1}} \frac{\partial^{2} C_{1}}{\partial y^{2}}$

$u \frac{\partial C_{2}}{\partial x}+v \frac{\partial C_{2}}{\partial y}=D_{S_{2}} \frac{\partial^{2} C_{2}}{\partial y^{2}}$,

and the appropriate $\mathrm{BC}$ 's are

$$
\begin{aligned}
& u=0, v=V_{w}(x), \quad C_{1}=C_{1 w}, \quad C_{2}=C_{2 w},-k \frac{\partial T}{\partial y}=h_{f}\left(T_{f}-T_{w}\right), \text { at } y=0, \\
& u \rightarrow U_{\infty}, v \rightarrow 0, C_{1} \rightarrow C_{1 \infty}, C_{2} \rightarrow C_{2 \infty}, T \rightarrow T_{\infty} \text { as } y \rightarrow \infty .
\end{aligned}
$$

Where, $V_{w}(x)<0$ and $V_{w}(x)>0$ represents suction and injection, respectively.

The rheological equation for an isotropic incompressible Casson fluid [25, 27] is defined as

$$
\tau_{i j}= \begin{cases}2\left(\mu_{B}+p_{y} / \sqrt{2 \pi}\right) e_{i j}, & \pi>\pi_{c} \\ 2\left(\mu_{B}+p_{y} / \sqrt{2 \pi_{c}}\right) e_{i j}, & \pi<\pi_{c}\end{cases}
$$

Introducing the following similarity transformation in order to express PDE's (1)-(6) in linear form

$$
\psi=\left(v x U_{\infty}\right)^{\frac{1}{2}} f(\eta), \quad \eta=y\left(\frac{U_{\infty}}{v x}\right)^{1 / 2}, \quad \theta(\eta)=\frac{T-T_{\infty}}{T_{f}-T_{\infty}}, \quad \phi_{1}(\eta)=\frac{C_{1}-C_{1 \infty}}{C_{1 w}-C_{1 \infty}}, \quad \phi_{2}(\eta)=\frac{C_{2}-C_{2 \infty}}{C_{2 w}-C_{2 \infty}},
$$

where, $\psi$ is the stream function, and the velocity components are given by

$$
u=U_{\infty} f^{\prime}(\eta), v=-\frac{1}{2} \sqrt{\frac{U_{\infty} v}{x}}\left[f-\eta f^{\prime}\right] \text {. }
$$

Substituting equations (8) and (9) in Eqn's. (1) - (6) to a set of ordinary differential equations in the non-dimensional form

$$
\left(1+\frac{1}{\beta}\right) f^{\prime \prime \prime}+\frac{1}{2} f f^{\prime \prime}-M^{2} f^{\prime}+\lambda\left[\theta+N_{1} \phi_{1}+N_{2} \phi_{2}\right]=0
$$


$\theta^{\prime \prime}+\frac{\operatorname{Pr}}{2} f \theta^{\prime}=0$

$\phi_{1}^{\prime \prime}+\frac{S c_{1}}{2} f \phi_{1}^{\prime}=0$

$\phi_{2}^{\prime \prime}+\frac{S c_{2}}{2} f \phi_{2}{ }^{\prime}=0$.

and the distorted boundary conditions are given by

$f(\eta)=f_{w}, f^{\prime}(\eta)=0, \theta^{\prime}(\eta)=-a[1-\theta(\eta)], \quad \phi_{1}(\eta)=1, \phi_{2}(\eta)=1$ at $\eta=0$,

$f^{\prime}(\eta)=1, \quad \theta(\eta)=0, \quad \phi_{1}(\eta)=0, \quad \phi_{2}(\eta)=0$ at $\eta=\eta_{\infty}$,

where, $\eta_{\infty}$ is the edge of the boundary layer; $M=B_{0} \sqrt{\sigma / \rho U_{\infty}}$ is the magnetic parameter; $\operatorname{Pr}=v / \alpha$ is the Prandtl number; $S c_{1}=v / D_{S_{1}}$ and $S c_{2}=v / D_{S_{2}}$ are the Schmidt numbers of the solutals $S_{m}(m=1,2)$ respectively; $\lambda=\lambda(x)$ is the mixed convection parameter and $N_{1}=N_{1}(x)$ and $N_{2}=N_{2}(x)$ are the buoyancy force parameters for concentration $C_{m}(m=1,2)$, which are given by

$\lambda(x)=\frac{G r_{x}}{\operatorname{Re}_{x}^{2}}, N_{1}(x)=\frac{G r_{x_{1}}}{\operatorname{Re}_{x}^{2}}, N_{2}(x)=\frac{G r_{x_{2}}}{\operatorname{Re}_{x}^{2}}$.

Also, $G r_{x}=g \beta_{T}\left(T_{f}-T_{\infty}\right) x^{3} / v^{2}, G r_{x_{1}}=g \beta_{C_{1}}\left(C_{1 w}-C_{1 \infty}\right) x^{3} / v^{2}$ and $G r_{x_{2}}=g \beta_{C_{2}}\left(C_{2 w}-C_{2 \infty}\right) x^{3} / v^{2}$ are local Grashof numbers, and $\operatorname{Re}_{x}=U_{\infty} x / v$ is the local Reynolds number. Mixed convection parameter corresponds to assisting flow, when $\lambda>0$, and opposing flow when $\lambda<0$. The skin friction coefficient $C_{f x}$, the Nusselt number $N u_{x}$, and the Sherwood numbers $S h_{x_{1}}, S h_{x_{2}}$ are defined as,

$$
\begin{aligned}
& C_{f x}=2 \operatorname{Re}_{x}^{-1 / 2} f^{\prime \prime}(0), \operatorname{Re}_{x}{ }^{-1 / 2} N u_{x}=-\theta^{\prime}(0), \\
& S h_{x_{1}}=-\operatorname{Re}_{x}^{-1 / 2} \phi_{1}^{\prime}(0), S h_{x_{2}}=-\operatorname{Re}_{x}^{-1 / 2} \phi_{2}^{\prime}(0) .
\end{aligned}
$$

\section{Results and Discussion}

To understand the physics of the model, the flow equations (10) - (13) are solved along (14) using the fourth-order Runge-Kutta integral scheme of shooting technique with the help of the Newton-Raphson method, and results are discussed through plots. This iterative process is repeated by choosing the value of $\eta$ sufficiently small at each time until the error becomes less than a predefined tolerance up to six decimal places of accuracy. In the present numerical study. The fluid properties such as velocity, temperature, concentration, skin friction as well as heat transfer rate are discussed for various non-dimensional parameters

$$
\begin{gathered}
f_{w}\left(-0.4 \leq f_{w} \leq 0.4\right), \lambda(1 \leq \lambda \leq 20), N_{1}\left(0.1 \leq N_{1} \leq 15.69\right), N_{2}\left(-1.36 \leq N_{2} \leq 1.36\right), \operatorname{Pr}(0.7 \leq \operatorname{Pr} \leq 25), \\
M(1 \leq M \leq 5), \beta(1 \leq \beta \leq 5), a(1 \leq a \leq 20), S c_{1}\left(649.19 \leq S c_{1} \leq 949.19\right), S c_{2}\left(1946.19 \leq S c_{2} \leq 4946.19\right)
\end{gathered}
$$

which are involved in the physical model from figures $2-12$. To verify the obtained results are correct or not, a comparative study has been done in Table.1 with the available literature [17] and [18] in the absence of few non-dimensional parameters and found good agreement.

Table 1. Comparison of $-\theta^{\prime}(0)$ for various values of $a$ when $\operatorname{Pr}=10, \mathrm{f}_{\mathrm{w}}=0, \mathrm{M}=0$ and $\lambda=0$.

\begin{tabular}{c|c|c|c}
$a$ & Aziz [17] & Ishak [18] & Present result \\
\hline 0.8 & 0.3812 & 0.381191 & 0.381201 \\
\hline 1 & 0.4213 & 0.421344 & 0.421252
\end{tabular}




\begin{tabular}{c|c|c|c}
\hline$a$ & Aziz[17] & Ishak [18] & Present result \\
\hline 5 & 0.6356 & 0.635583 & 0.635601 \\
\hline 10 & 0.6787 & 0.678721 & 0.678711 \\
\hline 20 & 0.7026 & 0.702563 & 0.702601
\end{tabular}

Table 2. Thermo physical properties of $\mathrm{NaCl}$ and Sucrose at $25^{\circ} \mathrm{C}$ [7].

\begin{tabular}{|c|c|c|c|c|c|c|c|}
\hline Components & Morality & Weighs\% & $v\left(\times 10^{-6}\right)$ & $D_{S}\left(\times 10^{-9}\right)$ & $S c$ & $\Delta C$ & $N c$ \\
\hline \multirow{5}{*}{$\mathrm{NaCl}$} & 0.01 & 0.0584 & 1.003 & 1.545 & 649.19 & 0.0108 & -2.48 \\
\hline & 0.05 & 0.2922 & 1.007 & 1.502 & 670.43 & 0.03 & -6.86 \\
\hline & 0.1 & 0.5844 & 1.011 & 1.483 & 681.72 & 0.05 & -11.43 \\
\hline & 0.5 & 2.922 & 1.031 & 1.472 & 700.4 & 0.4 & -91.51 \\
\hline & 1.0 & 5.844 & 1.058 & 1.484 & 712.93 & 0.5 & -114.3 \\
\hline \multirow{5}{*}{ Sucrose } & 0.01 & 0.342 & 1.014 & 0.521 & 1946.25 & 0.0108 & -1.36 \\
\hline & 0.05 & 1.7115 & 1.043 & 0.468 & 2226.4 & 0.03 & -3.77 \\
\hline & 0.1 & 3.423 & 1.08 & 0.451 & 2597.4 & 0.05 & -6.28 \\
\hline & 0.5 & 17.115 & 1.610 & 0.442 & 3635.95 & 0.4 & -50.3 \\
\hline & 1.0 & 34.23 & 3.535 & 0.466 & 7585.83 & 0.5 & -62.87 \\
\hline
\end{tabular}

The effect of the Schmidt number $\left(S c_{i}, i=1,2\right)$ on the concentration distribution $\left(\phi_{i}, i=1,2\right)$ are shown in Fig. 2(a) and 2(b). It is perceived that the magnitude of concentration profiles with the escalation of Schmidt numbers $\left(S c_{i}, i=1,2\right)$ for both $\phi_{1}(\eta)$ (Fig. 2(a)) and $\phi_{2}(\eta)$ (Fig.2(b)). The above behavior is observed since enhancing $S c$ the chemical molecular diffusivity, which ensues in less diffusion due to the mass transfer. The local Schmidt number values refer to the thickness of the large species' diffusion and hence to the rise in the thickness of the concentration boundary layer. It should be noticed that when combined with sucrose, the thickness of the species concentration boundary layer for $\mathrm{NaCl}$ is found to be greater since the sizes of the ions $\left(\mathrm{Na}^{+}\right.$and $\left.\mathrm{Cl}^{-}\right)$are smaller for the salt softened in water relative to Sucrose molecules dissolved in the water. As a consequence, $\mathrm{NaCl}$ diffuses deep into the water in comparison to sucrose.

Figure 3 portrays the effect of suction and injection on concentration profiles $\left(\phi_{1}(\eta)\right)$ for $N_{1}=0.1$ and $N_{2}=-1.36$. It can be seen that in the instance of injection, $\left(f_{w}<0\right)$ the liquid is passed away from the surface, instigating a decrease in concentration gradient as it stabs to continue the comparable concentration near the surface over a trifling region, and this effect is reversed in the case of suction $\left(f_{w}>0\right)$. It is observed that in an instance of suction $\left(f_{w}>0\right)$, the concentration boundary layer thickness declines, and in the case of injection $\left(f_{w}<0\right)$, concentration boundary layer thickness upturns. In particular, a roughly $43 \%$ decrease in boundary layer thickness is seen as $\operatorname{suction}\left(f_{w}>0\right)$ increases from $f_{w}=0.1$ to $f_{w}=0.2$. Whereas, approximately $20 \%$ increase in boundary layer thickness is observed as injection $\left(f_{w}<0\right)$ increases from $f_{w}=-0.1$ to $f_{w}=-0.2$. Similarly, the velocity profile depicted in Fig. 4 shows that suction $\left(f_{w}=0.3\right)$ decreases the velocity boundary layer thickness while injection $\left(f_{w}=-0.3\right)$ does the reverse. For example, from Fig.4 shows that the velocity boundary layer thickness $\left(\eta_{\infty}\right)$ for $\beta=5.0$ and $f_{w}=0.3$ is 6 whereas, $\eta_{\infty} \approx 7.5$ for $f_{w}=-0.3$. In the case of injection fluid, a reduction in the velocity gradient is admitted away from the surfa ce, and this effect is reversed in the case of suction. Injection decreases the sheerness of the wall's velocity profile, but the sheerness increases with suction. Furthermore, in Figure 4 that with the rise in the Casson parameter $\beta$, the velocity decreases. As increasing $\beta$, the fluid flow becomes more viscous, which resulting in fluid velocity reduction. Evan, as $\beta$ raises, the momentum boundary layer thickness reduces. The outcomes shown in Fig. 4 indicate an 
approximately $6.5 \%$ decrease in velocity as the Casson parameter upturns from $\beta=1.0$ to $\beta=5.0$ for $f_{w}=-0.3$.

Effects of Prandtl number (Pr), as well as the mixed convection parameteron the temperature and velocity profiles, are depicted in Fig.5 and Fig.6. Figure 5 that as the Prandtl number and the mixed convection parameter increase, the thermal boundary layer thickness reduces. Quantitatively, for $\lambda=3$ and $\operatorname{Pr}=10$, the thermal boundary layer thickness is approximately 1.6, whereas the thermal boundary layer thickness is approximately 1.25 for $\mathrm{Pr}$ $=21$. The fluid's thermal conductivity decreases with a rise in the value of Prandtl number, and hence thermal boundary layer thickness declines. Also, it can be observed that the effect of the Prandtl number decreases with the increase in the value of the buoyancy parameter. For example, approximately $1 \%$ decrease in thermal boundary layer thickness is observed as the Prandtl number increases from $\operatorname{Pr}=21$ to $\operatorname{Pr}=25 \lambda=21$, whereas about $7 \%$ decrease in the thermal boundary layer is observed $\lambda=3$. Fig. 6 shows that the buoyancy effect causes overreach in the velocity contour neat the wall for lower fluid of Prandtl number fluid ( $\operatorname{Pr}=7.0$ ), but the overreach is absent for the higher value of the fluid Prandtl number ( $\operatorname{Pr}=10)$. With the mixed convection parameter $(\lambda)$, the overshoot volume proliferates but decreases as the number of Prandtl number increases due to the stumpy viscosity of the fluid, as the added buoyancy force $(\lambda>0)$ functions as a positive pressure gradient, this increases the velocity inside the boundary layer. The overreach is absent for higher Prandtl number fluids because the added viscous fluid makes it less profound to mixed convection parameter $(\lambda)$.

The impact of the convective parameter $(a)$ on the temperature contour is displayed in Fig.7. It is noticed that as the convective parameter rises, the plate surface temperature increases. As the convective parameter $(a)$ tends to infinity, the solution is the typical solution for the persistent surface temperature. The outcomes can also be perceived from the BC (i.e., $\theta(0)=1$ as $a \rightarrow \infty)$. The effect of buoyancy force parameters $N_{1}$ and $N_{2}$ on different profiles are presented in Fig.8 to 10. The fraction of buoyancy constraints $\left(N_{i}, i=1,2\right)$ is cast-off to characterize buoyancy's simulated strength and solutal convection within the stream areas. It can be seen from Fig. 8 that the improvement in the buoyancy parameters causes a decrease in the thickness of the boundary layer. From Fig.9, similar behavior is observed for the thermal boundary layer, and an opposite trend is noticed from Fig.10 as increases buoyancy ratio parameters for concentration boundary layer thickness. The buoyancy parameters' positive and negative signs $\left(N_{i}, i=1,2\right)$ indicate that the buoyancy parameter caused by concentration and thermal gradient act in the same and opposite direction.

The effects of magnetic parameter $(M)$ and Casson Parameter $(\beta)$ on the skin friction coefficient $f^{\prime \prime}(0)$ with $f_{w}$ for different values are displayed in Fig.11. It is noticed that the local skin friction decreases as the magnetic parameter $(M)$ increases. Also observed that, for $a=20.0, \operatorname{Pr}=21, N_{2}=2.48, S c_{1}=649.19, S c_{2}=1946.19, \lambda=1.0, \beta=5.0, N_{1}=-1.36$ approximately $11 \%$ decrease in $f^{\prime \prime}(0)$ is noticed as $M$ increases from 1 to 2 for $f_{w}=-0.1$. Suction increases skin friction since the particles are drawn near the wall during suction and injection reduces skin friction since fluid particles are carried away from the wall. Approximately $41 \%$ increase in skin friction is noticed for an increase in $f_{w}$ from - 0.1 to 0.1 when $\mathrm{M}=1$ and $\beta=1$. Also, an increase in the Casson parameter $(\beta)$ causes a decrease in local skin friction coefficient, an average of $30 \%$ decrease in $f^{\prime \prime}(0)$ is observed as the Casson 
parameter $(\beta)$ increases from 1.0 to 5.0. Variation of heat transfer $\theta^{\prime}(0)$ with $a$ for different values of Prandtl number $(\operatorname{Pr})$ and $f_{w}$ is displayed in Fig.12. It can be observed that as the Prandtl number increases, the heat transfer at the wall upturns, thereby decreasing in the boundary layer. Also, an approximately $9 \%$ increase in heat transfer at the wall is noticed as $\mathrm{Pr}$ increases from $\operatorname{Pr}=7$ to $\operatorname{Pr}=10$ for $f_{w}=0.2$ and $a=1.5$. Further, the increase in the value of the convection parameter $a$ increases the surface temperature. It can be observed that the temperature gradient near the wall increases as increasing the convection parameter. Approximately $18 \%$ increase in heat transfer at the wall is noticed as $a$ increases from 1.3 to 1.5 for $f_{w}=0$ and $\operatorname{Pr}=10$.
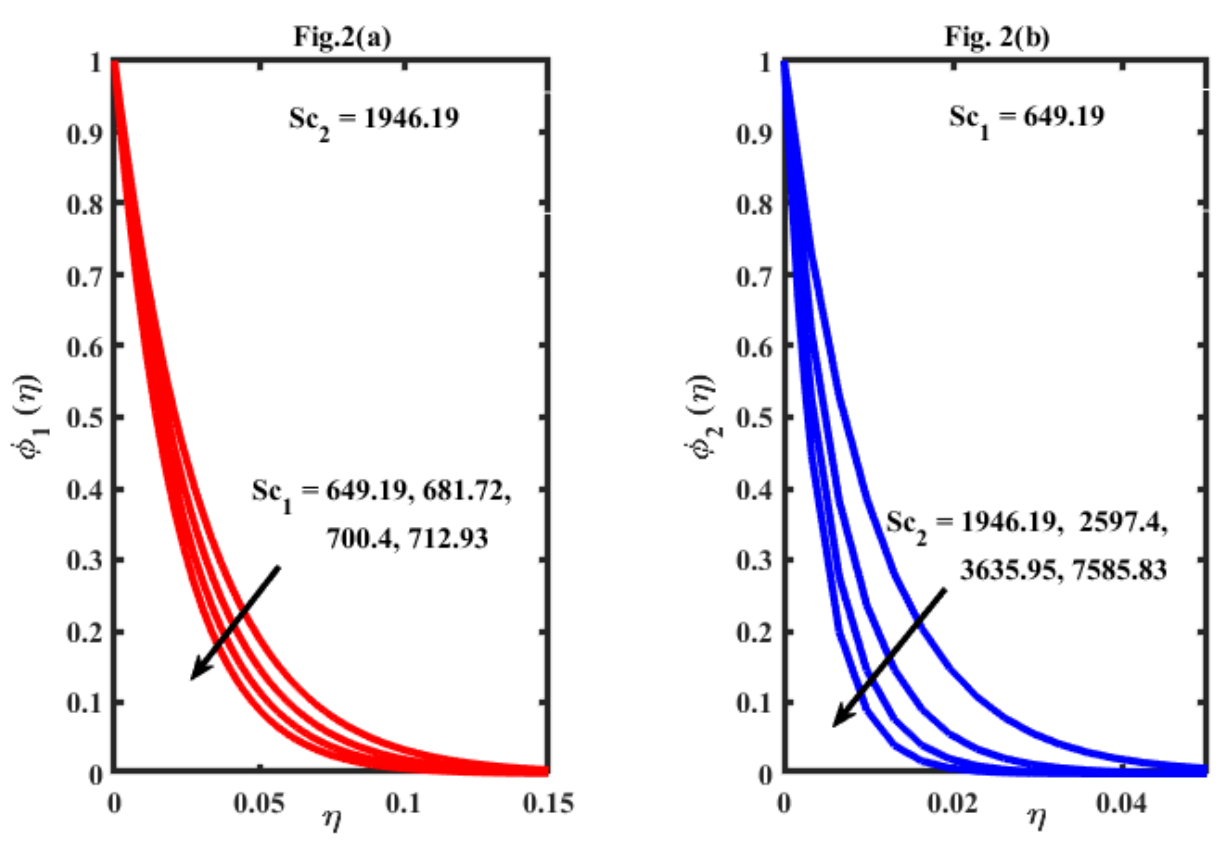

Figure 2. Impact of $S c_{1}$ and $S c_{2}$ on concentration profiles for $M=0.05, \operatorname{Pr}=7.0, a=3.0$,

$$
\beta=3.0, N_{2}=-1.36, N_{1}=0.1, f_{w}=0.1, \lambda=3.0 .
$$
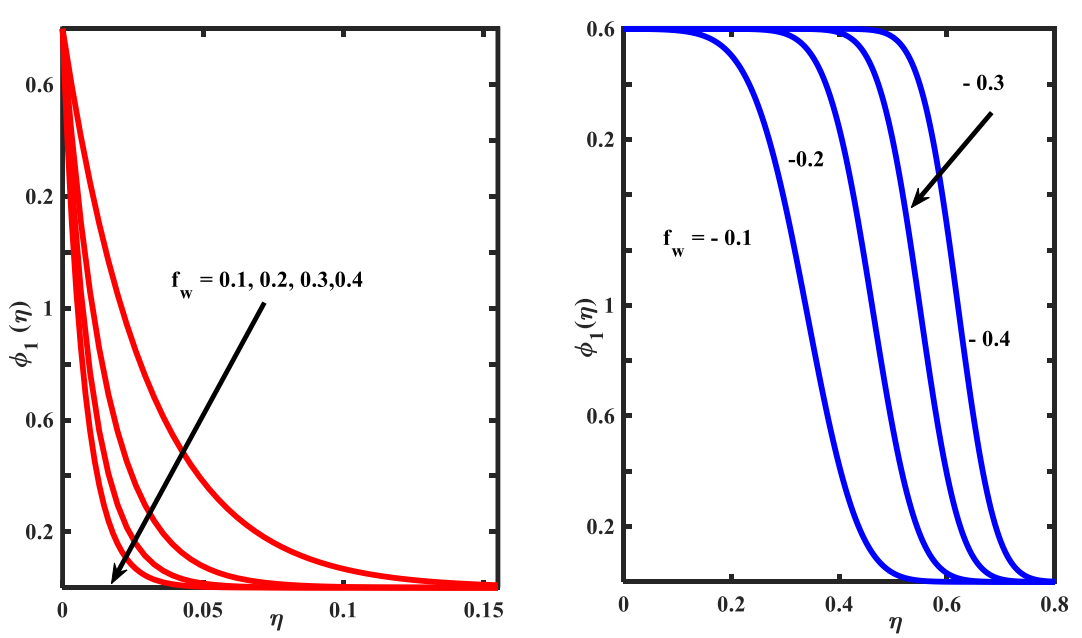

Figure 3. Effect of $f_{w}$ on concentration profile for $M=0.05, \operatorname{Pr}=7.0, a=3.0$, $\beta=3.0, S c_{1}=649.19, S c_{2}=1946.19, N_{2}=-1.36, N_{1}=0.1, f_{w}=0.1, \lambda=3.0$. 


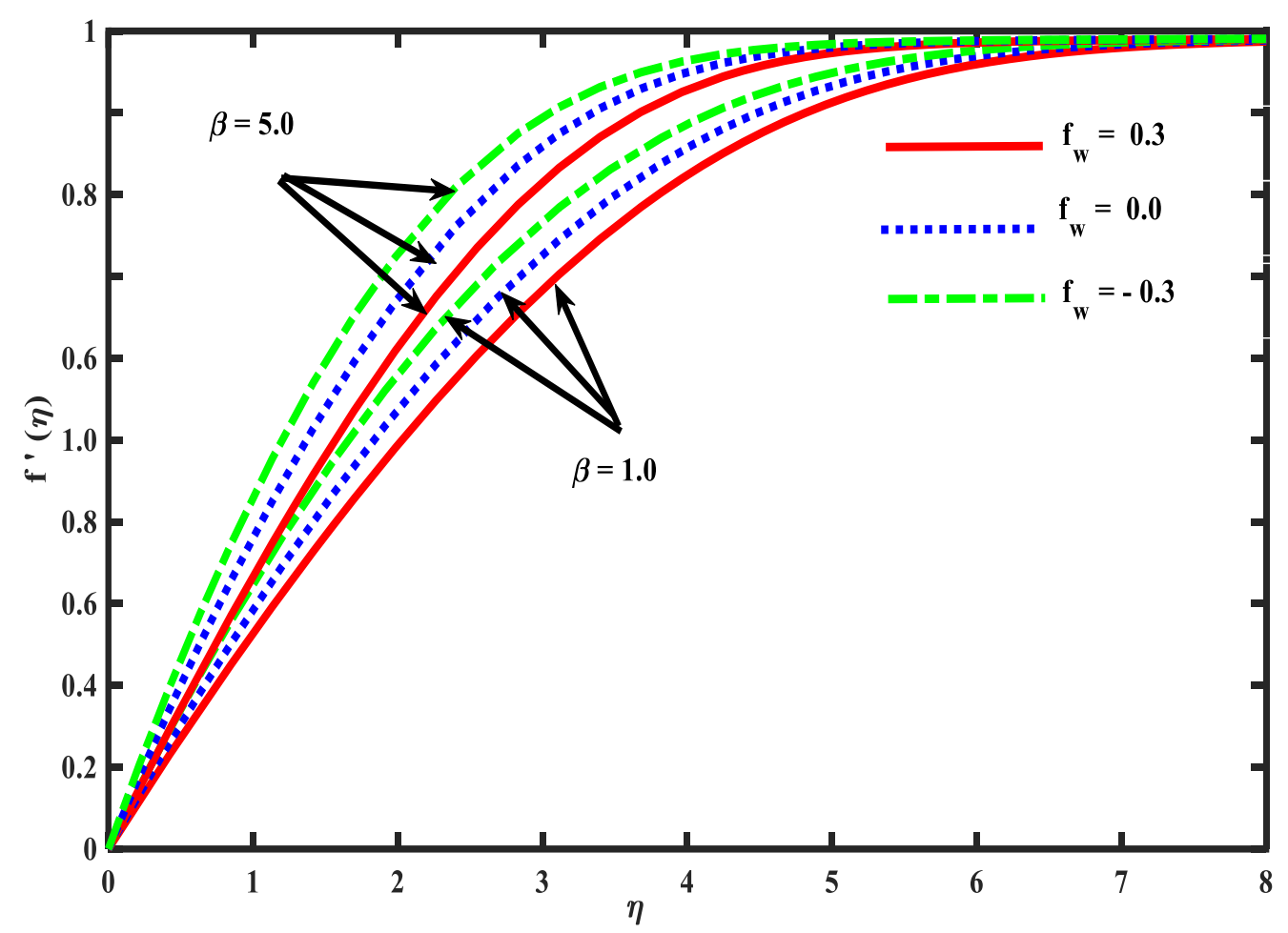

Figure 4. Impact of $\mathrm{f}_{\mathrm{w}}$ and $\beta$ on velocity profile for $M=0.05, \operatorname{Pr}=0.7, a=0.5$,

$$
S c_{1}=0.94, S c_{2}=1.66, N_{2}=0.3, N_{1}=0.1, \lambda=0.1 .
$$

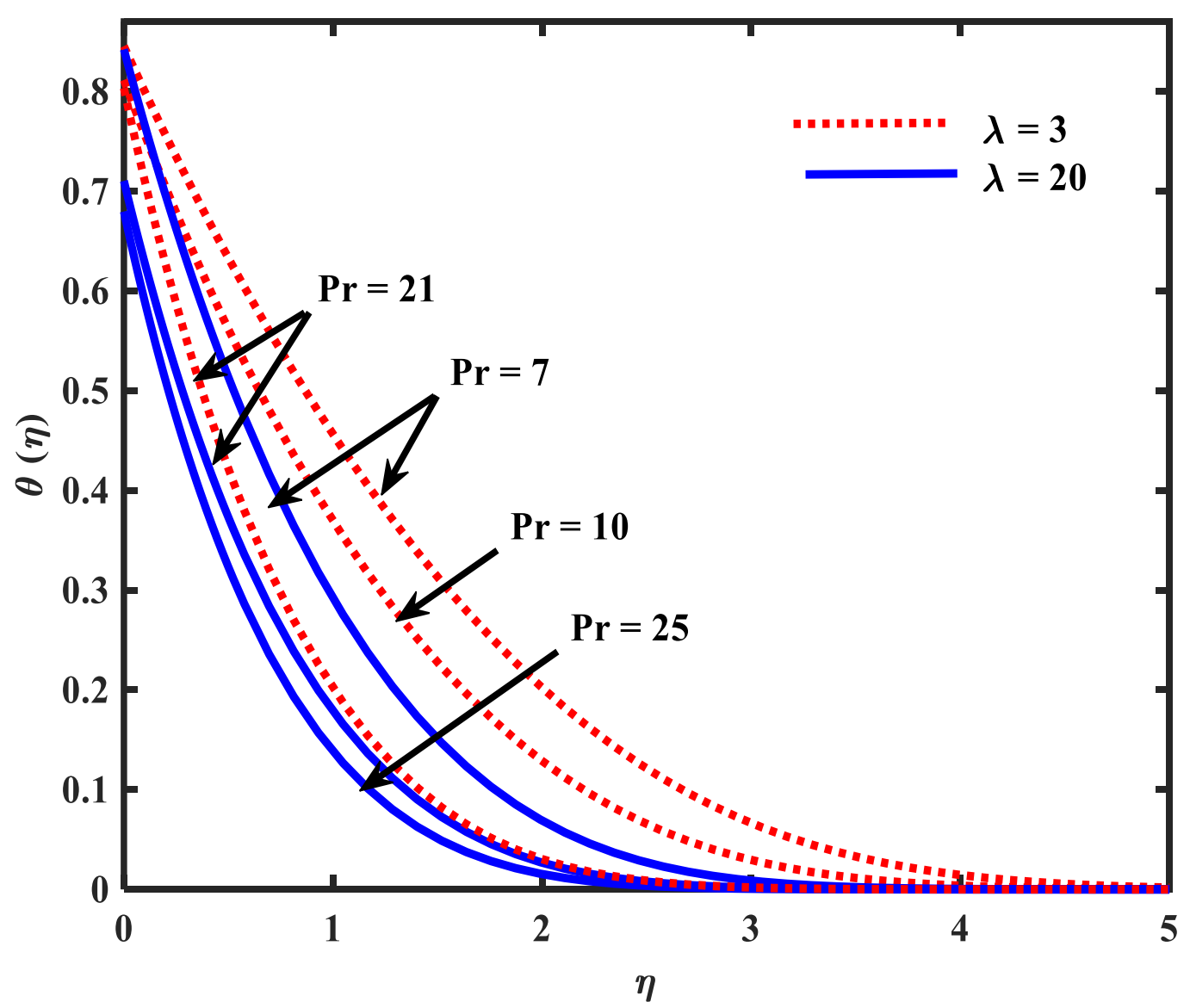

Figure 5. Impact of $\operatorname{Pr}$ and $\lambda$ on temperature profile for $M=0.05, f_{w}=0.1, a=3.0$,

$$
\beta=3.0, S c_{1}=649.19, S c_{2}=1946.19, N_{2}=-1.36, N_{1}=0.1 \text {. }
$$




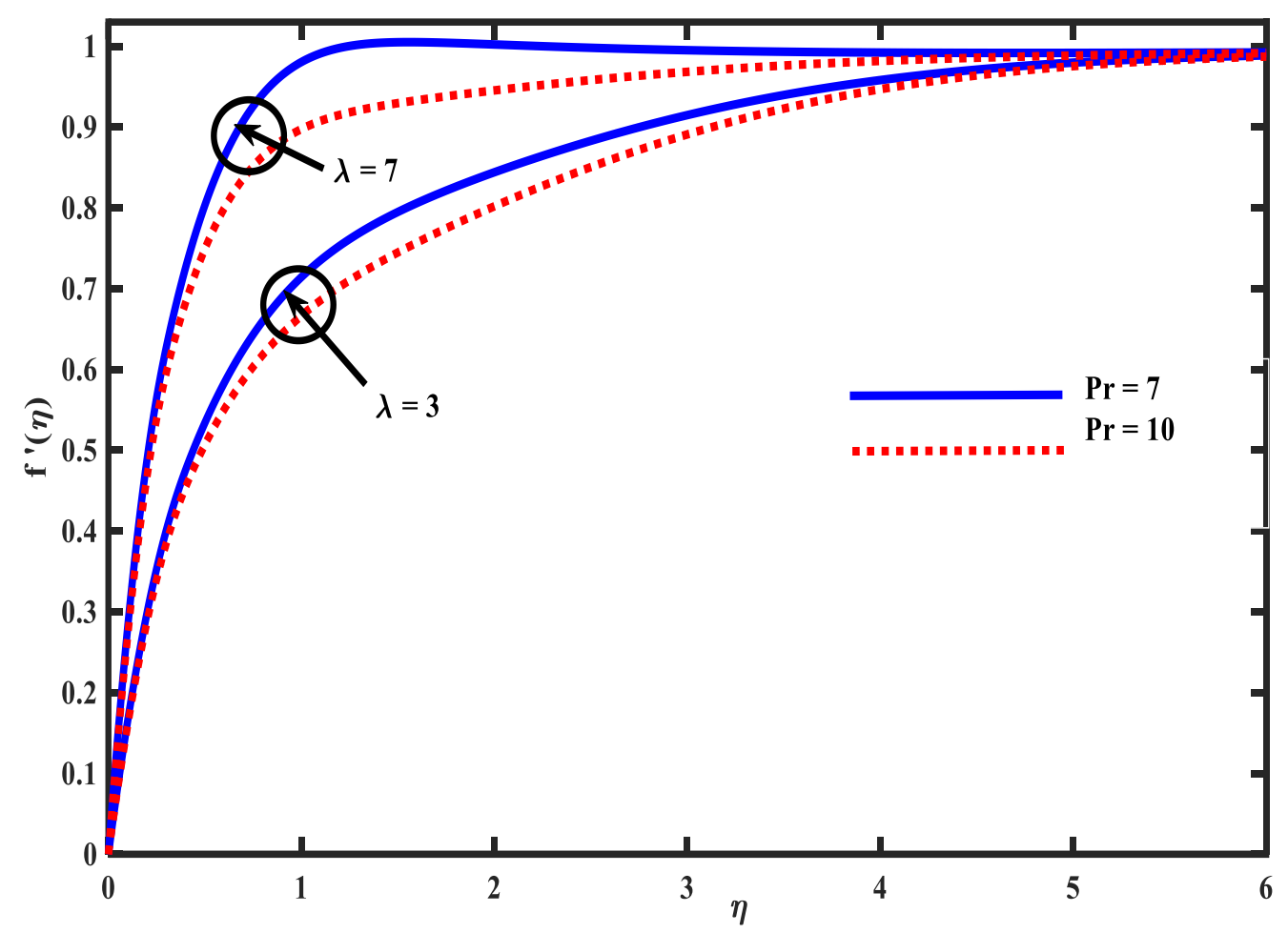

Figure 6. Impact of $\lambda$ and $\operatorname{Pr}$ on velocity profile for $M=0.05, a=20.0, \operatorname{Pr}=21$, $\beta=3.0, S c_{1}=649.19, S c_{2}=1946.19, N_{2}=1.36, N_{1}=-0.1, \lambda=3.0$.

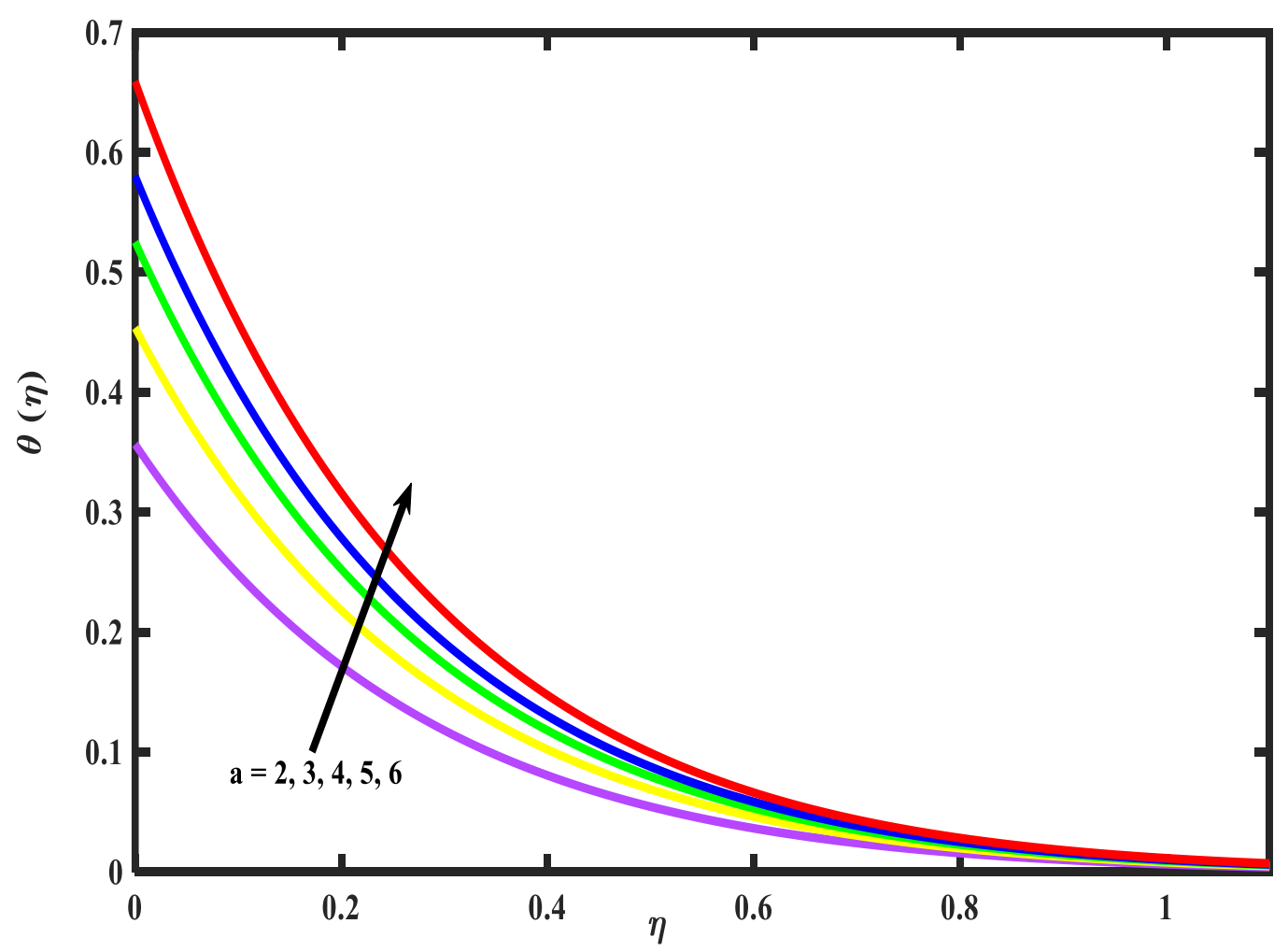

Figure 7. Effect of $a$ on temperature profile for $M=0.05, \operatorname{Pr}=7, a=20.0$, $S c_{1}=649.19, S c_{2}=1946.19, N_{1}=0.1, N_{2}=-1.36, \lambda=3.0, f_{w}=1$. 

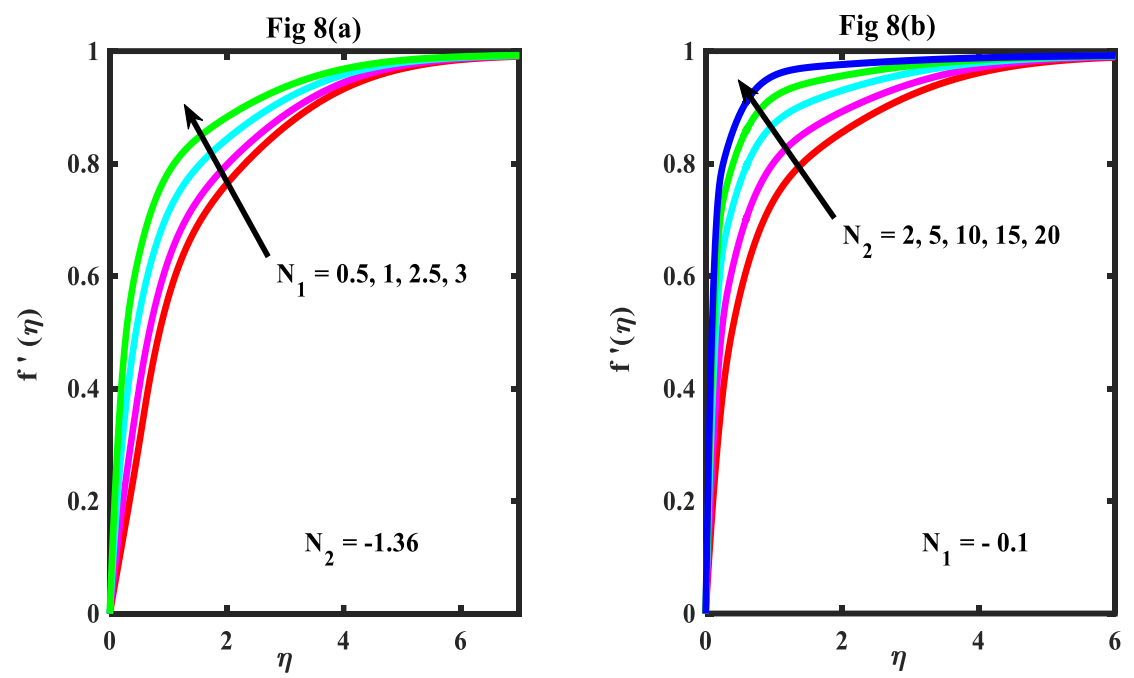

Figure 8. Effect of $N_{1}$ and $N_{2}$ on velocity profile for $M=0.05, \operatorname{Pr}=7, a=20.0$, $S c_{1}=649.19, S c_{2}=1946.19, N_{1}=0.1, N_{2}=-1.36, \lambda=3.0, f_{w}=-0.1, \beta=1.0$.
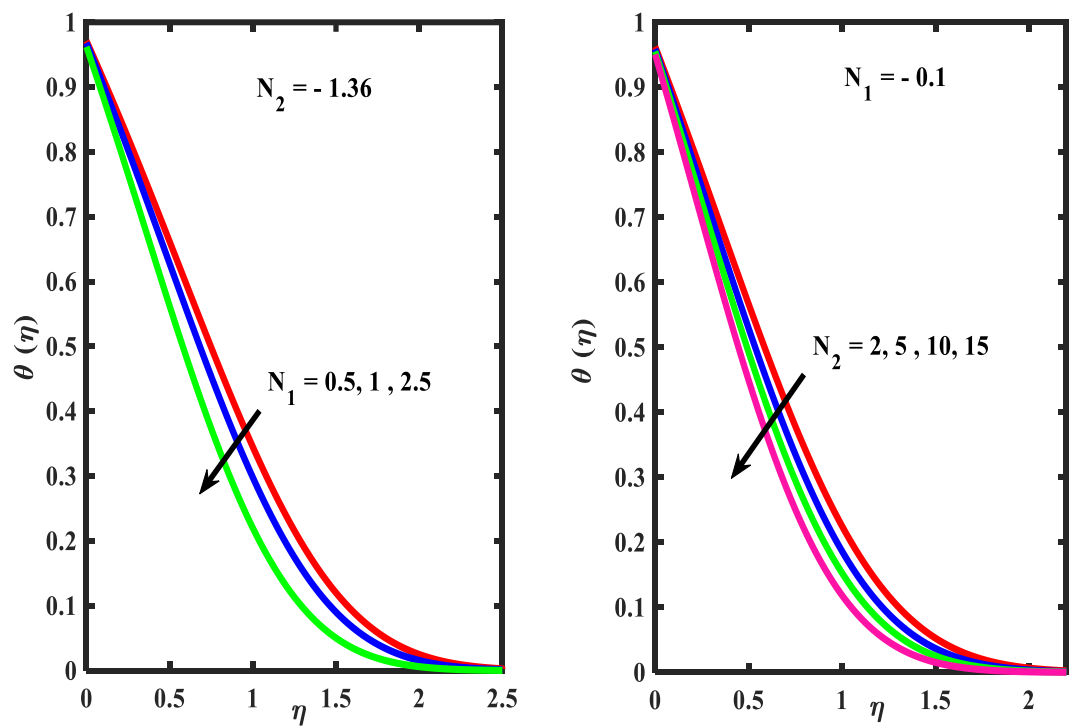

Figure 9. Effect of $N_{1}$ and $N_{2}$ on temperature profile for $M=0.05, \operatorname{Pr}=7, a=20.0$, $S c_{1}=649.19, S c_{2}=1946.19, \beta=1.0, \lambda=3.0, f_{w}=-0.1$.
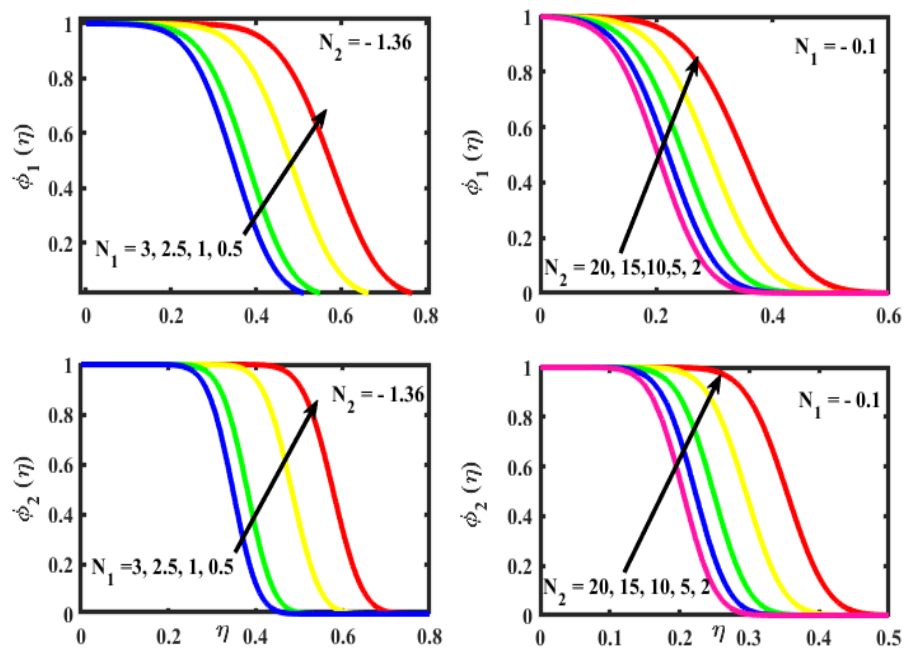

Figure 10. Effect of $N_{1}$ and $N_{2}$ on concentration profile for $M=0.05, \operatorname{Pr}=7, a=20.0$,

$$
S c_{1}=649.19, S c_{2}=1946.19, \beta=1.0, \lambda=3.0, f_{w}=-0.1 \text {. }
$$




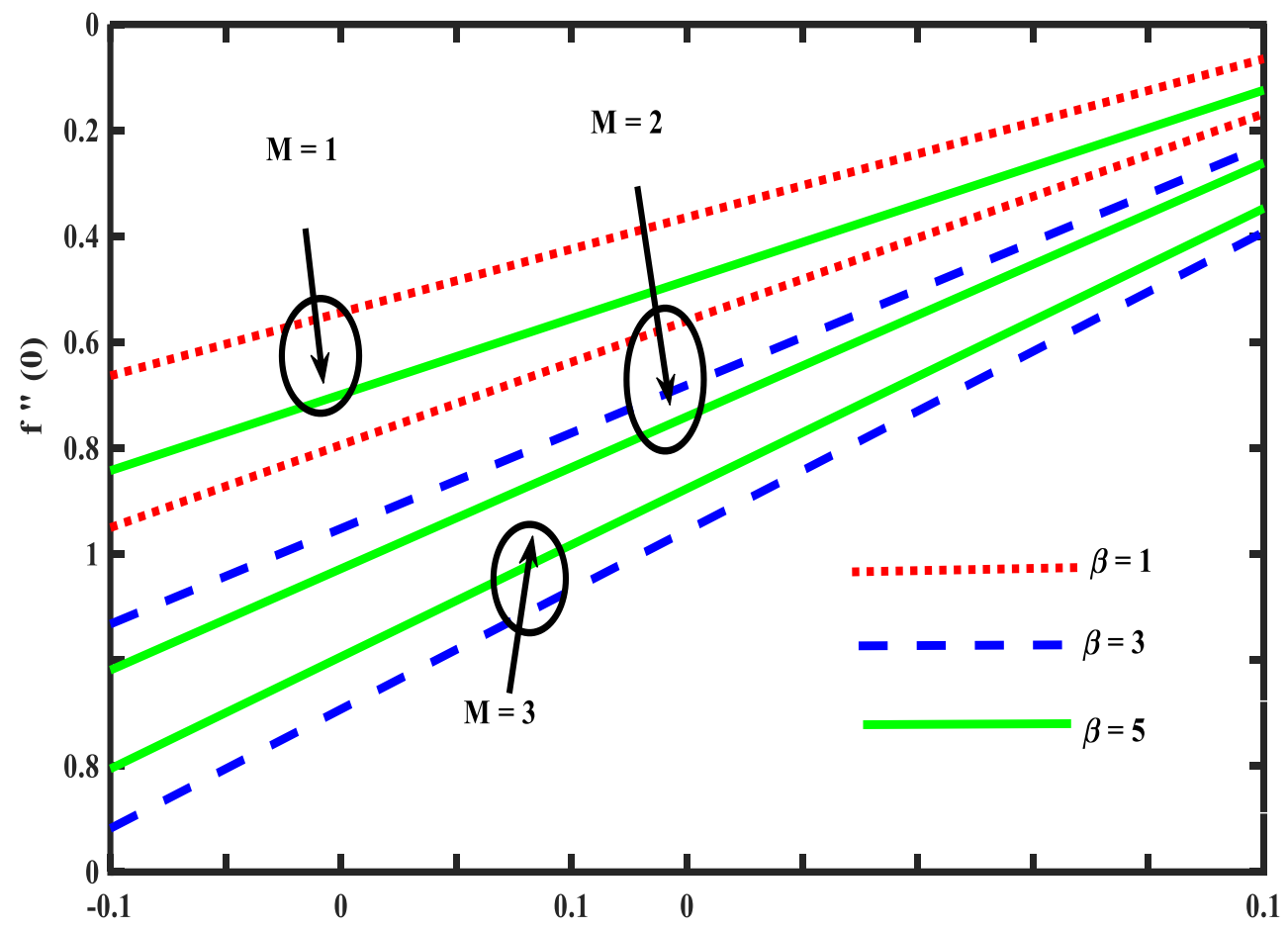

Figure 11. Deviation of $f^{\prime \prime}(0)$ with $f_{w}$ for $M$ and $\beta$ when $\operatorname{Pr}=21, a=20.0$, $S c_{1}=649.19, S c_{2}=1946.19, \lambda=1.0, N_{1}=-1.36$.

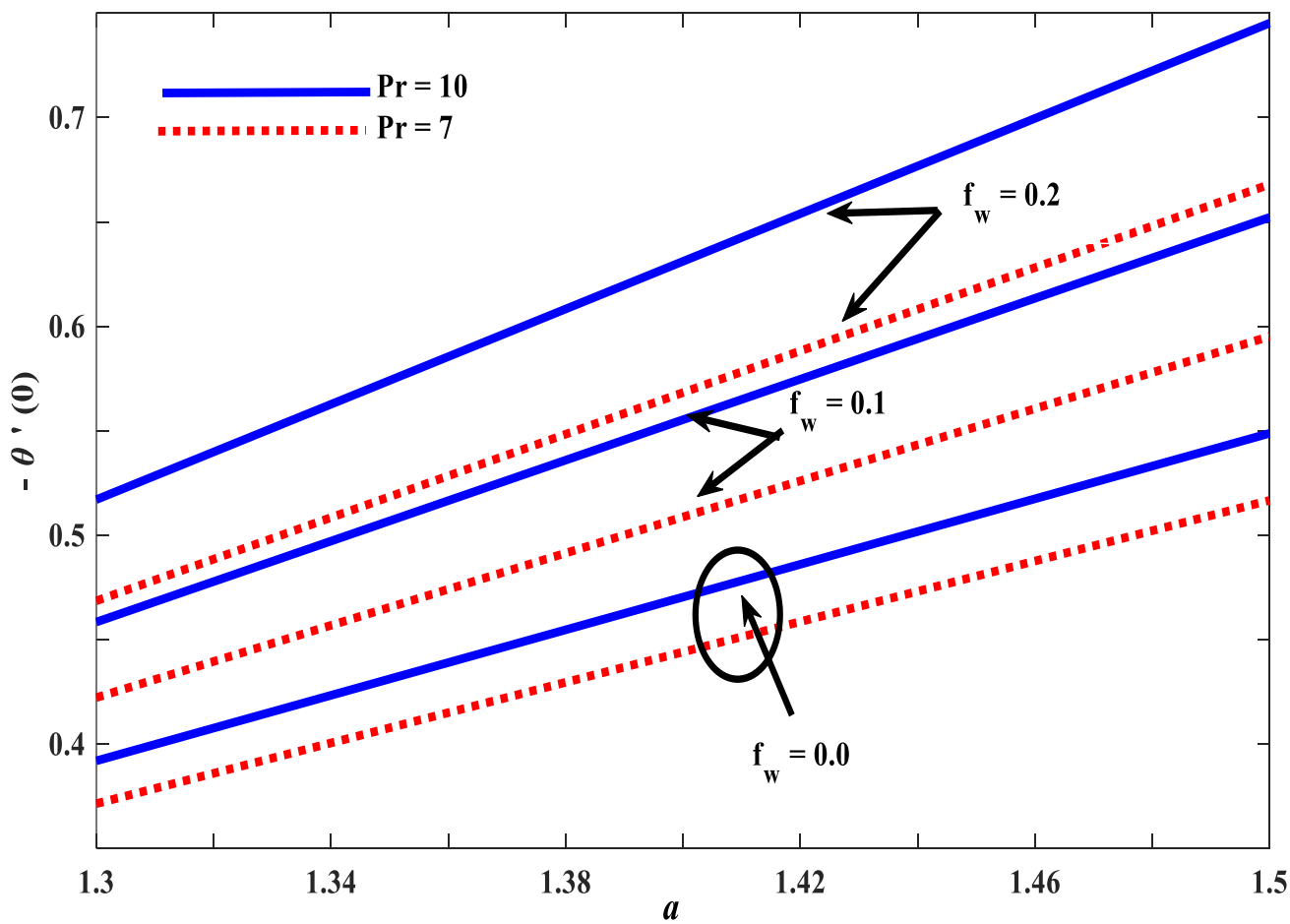

Figure 12. Variation of $-\theta^{\prime}(0)$ with $a$ for $\operatorname{Pr}$ and $\mathrm{f}_{\mathrm{w}}$ when $M=0.05, S c_{1}=649.19, S c_{2}=1946.19, \beta=1.0, \lambda=3.0, N_{1}=0.1, N_{1}=-1.36$.

\section{Conclusions}

The conclusions of the numerical study are as follows: (i) $\mathrm{NaCl}$ concentration boundary layer is thicker than that of sucrose. $\mathrm{NaCl}$ diffuses deeper into fluid than sucrose due to the smaller size of ions in $\mathrm{NaCl}$; (ii) Average of $6 \%$ decreases in velocity is noticed with the upturn of Casson parameter from $\beta=1.0$ to $\beta=5.0$.(iii) Rise in the value of mixed convection parameter $(\lambda)$ reduces the boundary layer thickness of both velocity, and temperature profile. 
Also, the effect $\lambda$ reduces with the rise in the Prandtl number; (iv) Skin friction at the wall reduces the proliferation in Casson parameter and Magnetic parameter; (v) The convection parameter $a$ increases the surface temperature. An average of $20 \%$ growth in heat transfer is noticed at the wall as $a$ it increases from 1.3 to 1 .

\section{Funding}

This research received no external funding.

\section{Acknowledgments}

The authors would like to thank the anonymous reviewers for their valuable comments and suggestions to improve the paper's quality.

\section{Conflicts of Interest}

The authors declare no conflict of interest.

\section{References}

1. Nield and Bejan, Convection in porous media, 4th edition, Springer verlag, 2013.

2. Kumari, M.; Nath, G. Doubly diffusive unsteady mixed convection flow over a vertical plate embedded in a porous medium. International Journal of Energy Research 1989, 13, 419-430, https://doi.org/10.1002/er.4440130406.

3. Mamou, M.; Bilgen, E.; Gobin, D.; Vasseur, P. Double-diffusive convection in an inclined slot filled with porous medium. European Journal of Mechanics B, Fluids 1995, 14, 629-652.

4. Patil, P.; Roy, S.; Chamkha, A. Double diffusive mixed convection flow over a moving vertical plate in the presence of internal heat generation and a chemical reaction. Turkish Journal of Engineering and Environmental Sciences 2009, 33, 193-205, https://doi.org/10.3906/muh-0905-21.

5. Murthy, P.V.S.N.; Sutradhar, A.; RamReddy, C. Double-Diffusive Free Convection Flow Past an Inclined Plate Embedded in a Non-Darcy Porous Medium Saturated with a Nanofluid. Transport in Porous Media 2013, 98, 553-564, https://doi.org/10.1007/s11242-013-0160-z.

6. Bandaru, M. Double Diffusive Convection of a Rotating Fluid Over a Vertical Plate Embedded in DarcyForchheimer Porous Medium with Non-Uniform Heat Sources. International Journal of Emerging Trends in Engineering and Development 2013, 2, 415-432.

7. Suresh Babu, R.; Bangalore, R.; P.A, D. Effects of variable fluid properties on a double diffusive mixed convection viscous fluid over a semi infinite vertical surface in a sparsely packed medium. Frontiers in Heat and Mass Transfer 2018, 10.

8. Rionero, S. Triple diffusive convection in porous media. Acta Mechanica 2013, 224, 447-458, https://doi.org/10.1007/s00707-012-0749-2.

9. Khan, W.A.; Culham, J.R.; Khan, Z.H.; Pop, I. Triple diffusion along a horizontal plate in a porous medium with convective boundary condition. International Journal of Thermal Sciences 2014, 86, 60-67, https://doi.org/10.1016/j.ijthermalsci.2014.06.035.

10. Raghunatha, K.R.; Shivakumara, I.S. Stability of triple diffusive convection in a viscoelastic fluid-saturated porous layer. Applied Mathematics and Mechanics 2018, 39, 1385-1410, https://doi.org/10.1007/s10483018-2376-8.

11. Patil, P.M.; Shashikant, A.; Momoniat, E.; Harley, C. Numerical simulation of unsteady triple diffusive mixed convection in NaCl-water and Sucrose-water solutions. International Journal of Heat and Mass Transfer 2018, 126, 147-155, https://doi.org/10.1016/j.ijheatmasstransfer.2018.05.166.

12. Patil, P.M.; Roy, M.; Roy, S.; Momoniat, E. Triple diffusive mixed convection along a vertically moving surface. International Journal of Heat and Mass Transfer 2018, 117, 287-295, https://doi.org/10.1016/j.ijheatmasstransfer.2017.09.106.

13. Samuel, N. Triple diffusive flow along a stretching sheet with variable thickness in a porous medium. Acta Technica CSAV (Ceskoslovensk Akademie Ved) 2018, 63, 407-422.

14. Umavathi, J.C.; Ali, H.M.; Patil, S.L. Triple diffusive mixed convection flow in a duct using convective boundary conditions. Mathematical Methods in the Applied Sciences 2020, 43, 9223-9244, https://doi.org/10.1002/mma.6617. 
15. Raghunatha, K.R.; Shivakumara, I.S.; Shankar, B.M. Weakly non-linear stability analysis of triple diffusive convection in a Maxwell fluid saturated porous layer. Applied Mathematics and Mechanics 2018, 39, 153168, https://doi.org/10.1007/s10483-018-2298-6.

16. Khan, Z.H.; Khan, W.A.; Sheremet, M.A. Enhancement of heat and mass transfer rates through various porous cavities for triple convective-diffusive free convection. Energy 2020, 201, https://doi.org/10.1016/j.energy.2020.117702.

17. Khan, Z.H.; Khan, W.A.; Tang, J.; Sheremet, M.A. Entropy generation analysis of triple diffusive flow past a horizontal plate in porous medium. Chemical Engineering Science 2020, 228, https://doi.org/10.1016/j.ces.2020.115980.

18. Nawaz, M.; Awais, M. Triple diffusion of species in fluid regime using tangent hyperbolic rheology. Journal of Thermal Analysis and Calorimetry 2020, https://doi.org/10.1007/s10973-020-10026-0.

19. Mohamed Isa, S.S.P.; Arifin, N.M.; Nazar, R.; Bachok, N.; Ali, F.M. The effect of convective boundary condition on MHD mixed convection boundary layer flow over an exponentially stretching vertical sheet. Journal of Physics: Conference Series 2017, 5, 949-964, https://doi.org/10.1088/1742-6596/949/1/012016.

20. Sharada, K.; Shanker, B. MHD Mixed Convection Flow of a Casson Fluid over an Exponentially Stretching Surface with the Effects of Soret, Dufour, Thermal Radiation and Chemical Reaction. World Journal of Mechanics 2015, 05, 165-177, https://doi.org/10.4236/wjm.2015.59017.

21. Hayat, T.; Shehzad, S.A.; Alsaedi, A.; Alhothuali, M.S. Mixed Convection Stagnation Point Flow of Casson Fluid with Convective Boundary Conditions. Chinese Physics Letters 2012, 29, https://doi.org/10.1088/0256-307x/29/11/114704.

22. John, E.C.; Yakubu, S.I.; Abe-I-Kpeng, G. MHD flow of Casson fluid over a vertical plate embedded inporous media with joule heating and convective boundary condition. Asian Journal of Mathematics and Computer Resources 2017, 19, 50-64.

23. Raju, R.S.; Reddy, B.M.; Reddy, G.J. Influence of Angle of Inclination on Unsteady MHD Casson Fluid Flow Past a Vertical Surface Filled by Porous Medium in Presence of Constant Heat Flux, Chemical Reaction and Viscous Dissipation. Journal of Nanofluids 2017, 6, 668-679, https://doi.org/10.1166/jon.2017.1368.

24. Muthuraj, R.; Selvi, R.K.; Srinivas, S. Thermodynamic analysis on MHD Casson nanofluid flow in a vertical porous space with stretching walls. Global Journal of Researches in Engineering 2018, 18, 1-15.

25. Kataria, H.; Patel, H. Heat and mass transfer in magnetohydrodynamic (MHD) Casson fluid flow past over an oscillating vertical plate embedded in porous medium with ramped wall temperature. Propulsion and Power Research 2018, 7, 257-267, https://doi.org/10.1016/j.jppr.2018.07.003.

26. Saqib, M.; Ali, F.; Khan, I.; Sheikh, N.A. Heat and mass transfer phenomena in the flow of Casson fluid over an infinite oscillating plate in the presence of first-order chemical reaction and slip effect. Neural Computing and Applications 2018, 30, 2159-2172, https://doi.org/10.1007/s00521-016-2810-x.

27. Khan, A.; Khan, I.; Khan, A.; Shafie, S. Heat Transfer Analysis in MHD Flow of Casson Fluid Over a Vertical Plate Embedded in a Porous Medium with Arbitrary Wall Shear Stress. Journal of Porous Media 2018, 21, https://doi.org/10.1615/JPorMedia.2018018872.

28. Bhaskar, C.; Sheri, S.; Suram, A. Numerical analysis of MHD casson fluid flow over an exponentially accelerated vertical plate in embedded porous medium with ramped wall temperature and ramped surface concentration in uniform magnetic field. International Journal of Applied Power Engineering (IJAPE) 2020, 9, 89-99, http://doi.org/10.11591/ijape.v9.i2.pp89-99.

29. Sumera, D.; Azizah M.R.; Azizan, S. Triple solutions and stability analysis of mixed convection boundary flow of Casson nanofluid over an exponentially vertical stretching/shrinking sheet. Journal of Advanced Research in Fluid Mechanics and Thermal Sciences 2020, 72, 94-110, https://doi.org/10.37934/arfmts.72.1.94110.

30. Manjappa, A.; Jayanna, G.B.; Chandrappa, P.B. Triple diffusive flow of Casson nanofluid with buoyancy forces and non-linear thermal radiation over a horizontal plate. Archives of Thermodynamics 2019, 40, 4969, https://doi.org/10.24425/ather.2019.12828.

31. Rao, P.S.; Prakash, O.; Mishra, S.R.; Sharma, R.P. Similarity solution of three-dimensional MHD radiative Casson nanofluid motion over a stretching surface with chemical and diffusion-thermo effects. Heat Transfer 2020, 49, 1842-1862, https://doi.org/10.1002/htj.21696.

32. Lund, L.A.; Omar, Z.; Khan, I.; Raza, J.; Sherif, E.-S.M.; Seikh, A.H. Magnetohydrodynamic (MHD) Flow of Micropolar Fluid with Effects of Viscous Dissipation and Joule Heating Over an Exponential Shrinking Sheet: Triple Solutions and Stability Analysis. Symmetry 2020, 12, https://doi.org/10.3390/sym12010142.

33. Ramanna, S.; Moss, H.G.; McKinnon, E.T.; Yacoub, E.; Helpern, J.A.; Jensen, J.H. Triple diffusion encoding MRI predicts intra-axonal and extra-axonal diffusion tensors in white matter. Magnetic Resonance in Medicine 2020, 83, 2209-2220, https://doi.org/10.1002/mrm.28084. 